\title{
Dopamine Modulates Reward System Activity During Subconscious Processing of Sexual Stimuli
}

\author{
Nicole YL Oei*, 1,2 , Serge ARB Rombouts ${ }^{1,2,3}$, Roelof P Soeter ${ }^{1,2,3}$, Joop M van Gerven ${ }^{4}$ and Stephanie Both ${ }^{1,5}$ \\ 'Leiden Institute for Brain and Cognition (LIBC), Leiden University, Leiden, The Netherlands; ' Department of Radiology, Leiden University Medical \\ Center, Leiden, The Netherlands; ${ }^{3}$ Institute of Psychology, Leiden University, Leiden, The Netherlands; ${ }^{4}$ Centre for Human Drug Research, Leiden, \\ The Netherlands; ${ }^{5}$ Department of Gynaecology, Leiden University Medical Center, Leiden, The Netherlands
}

\begin{abstract}
Dopaminergic medication influences conscious processing of rewarding stimuli, and is associated with impulsive-compulsive behaviors, such as hypersexuality. Previous studies have shown that subconscious subliminal presentation of sexual stimuli activates brain areas known to be part of the 'reward system'. In this study, it was hypothesized that dopamine modulates activation in key areas of the reward system, such as the nucleus accumbens, during subconscious processing of sexual stimuli. Young healthy males $(n=53)$ were randomly assigned to two experimental groups or a control group, and were administered a dopamine antagonist (haloperidol), a dopamine agonist (levodopa), or placebo. Brain activation was assessed during a backward-masking task with subliminally presented sexual stimuli. Results showed that levodopa significantly enhanced the activation in the nucleus accumbens and dorsal anterior cingulate when subliminal sexual stimuli were shown, whereas haloperidol decreased activations in those areas. Dopamine thus enhances activations in regions thought to regulate 'wanting' in response to potentially rewarding sexual stimuli that are not consciously perceived. This running start of the reward system might explain the pull of rewards in individuals with compulsive reward-seeking behaviors such as hypersexuality and patients who receive dopaminergic medication.

Neuropsychopharmacology (2012) 37, 1729-1737; doi:10.1038/npp.2012.19; published online 7 March 2012
\end{abstract}

Keywords: dopamine; backward masking; sexual motivation; functional imaging; reward system

\section{INTRODUCTION}

Having sexual desires carries important reproductive benefit, but when sexual urges become excessive, as in hyperactive sexual desire, this can result in sexual risk taking or sexually abusive behavior. Hyperactive sexual desire can cause marked personal distress and marital discord, and hypersexuality is a serious social problem when it develops into sexually abusive behavior. Very little is known about causes of hyperactive sexual desire, and empirically validated treatments are lacking. Insight into the underlying mechanisms of sexual motivation is essential to understand hypersexuality, and is needed to guide prevention as well as psychological and/or pharmacological treatment thereof.

According to incentive motivation models, sexual motivation is the result of the activation of a sexual response system by sexual stimuli (incentives) (Singer and Toates,

\footnotetext{
*Correspondence: Dr NYL Oei, Department of Radiology, Leiden Institute for Brain and Cognition (LIBC), Postzone C2-S, PO Box 9600 , Leiden 2300 RC, The Netherlands, Tel: + 3171526 4972; Fax: + 31 7| 524 8256, E-mail: N.Y.L.Oei@lumc.nl

Received II November 201 I; revised 3 February 2012; accepted 7 February 2012
}

1987; Stewart, 1995; Agmo, 1999; Both et al, 2007). Sensitivity of the sexual system, moderated by neurotransmitters and hormone levels in the body and the brain, is necessary for sexual motivation to emerge. As incentive motivation models imply, sexual stimuli play an essential role in eliciting sexual desire and behavior. A crucial question is, especially in a society that is flooded with sexual cues, what is it that makes sexual stimuli lead to exaggerated wanting or craving in some but not others?

One important neurobiological link was made between aberrant sexual reward-seeking behavior and dopamine (DA) after the discovery that dopaminergic therapy in Parkinson's disease can result in hypersexuality, and in abnormal stereotypical behaviors like excessive shopping, punding, or gambling (Evans et al, 2009). The impulsivecompulsive behaviors in patients treated for Parkinson's disease are hypothesized to reflect dopaminergic sensitization to rewards, similar to that proposed for drug addiction (Robinson and Berridge, 1993). Conscious processing of both primary rewards such as food and sex, but also of secondary rewards such as money, is usually associated with enhanced activations in the nucleus accumbens (NAcc), the central brain structure of the 'reward system'. This system is driven by the release of DA into the NAcc (Kringelbach and Berridge, 2009), which is thought to be involved in tagging 
stimuli with an incentive salience, making these stimuli 'wanted' (Berridge and Robinson, 1998). The incentive sensitization theory posits that as a result of dopaminergic neural adaptations, the DA system becomes hyper sensitive to rewarding stimuli, which in turn become hypersalient, not by increased liking, but by increased 'wanting'. Consistent with this theory, DA release in the ventral striatum following exposure to reward-related cues was higher in Parkinson patients with impulsive-compulsive behaviors, compared with Parkinson patients without impulsive-compulsive behaviors, which also suggests an individual vulnerability to becoming sensitized (O'Sullivan et al, 2011).

Dopaminergic modulation of conscious reward processing has been reported (Pessiglione et al, 2006; Pleger et al, 2009). 'Wanting', however, is not a consciously experienced wanting, but refers to the underlying implicit motivation process that drives behavior towards the rewarding target (Berridge and Robinson, 2003). Implicit reward processes can be assessed, and are of great informational value as they are unbiased by cognitive incentive, that is, subjective liking or disliking, desires, shame, positive, or negative outcome evaluations, which is especially relevant when presenting stimuli with sexual content. Typically, the reward system already responds to potentially rewarding sex- and drug-related stimuli that are presented outside awareness (Childress et al, 2008; Gillath and Canterberry, 2011). However, it is unknown whether DA modulates implicit motivation for sexual reward, at its earliest onset, outside awareness.

In this study, we therefore investigated whether DA modulates the response of the reward system to subconsciously processed sexual stimuli. We expected that increasing DA levels with a DA agonist would enhance, whereas inhibiting dopaminergic tone with a DA antagonist would decrease the activity in brain regions of the reward system, especially in the NAcc, caudate, insula, thalamus, orbitofrontal cortex (OFC), and dorsal anterior cingulate (dACC) (Haber and Knutson, 2010).

\section{MATERIALS AND METHODS}

\section{Participants}

Healthy, sexually active male volunteers from the general population were recruited by means of advertisements. The eligibility criteria were: no current (or history of) sexual complaints as determined by the International Index of Erectile Function (IIEF; Rosen et al, 1997) or psychiatric problems as determined by the Amsterdam Biographical interview (Wilde, 1963) and the MINI International Neuropsychiatric Interview (MINI; Sheehan et al, 1998); a heterosexual orientation, and no history of sexual abuse; no medical illness (or medical history), indicating a risk in using haloperidol or levodopa (eg, cardiac illness, depression, thyroid disorders, glaucoma); no use of medication affecting sexual response; and no current or recent use ( $<12$ weeks before participation) of psycho-pharmacological medication, psychotropic drugs, or medication that might interfere with haloperidol or levodopa (eg, cannabis or cocaine).

A total of 55 participants were included and randomly assigned to one of three experimental groups (L-dopa, haloperidol, or placebo) in a randomized, double-blind experimental design. Participants received a fixed dose of $100 \mathrm{mg}$ levodopa combined with $25 \mathrm{mg}$ of carbidopa (Sinemet, $T_{\max }=45 \mathrm{~min}$, half-time $=1-2 \mathrm{~h} ;$ Sagar and Smyth, 2000; Khor and Hsu, 2007), or haloperidol (3 mg, $T_{\max }=3-6 \mathrm{~h}$, half-time $=14-36 \mathrm{~h}$; Midha et al, 1989; LiemMoolenaar et al, 2010), or placebo. A PET study in healthy volunteers demonstrated that a single dose of Sinemet changes DA levels in the putamen and caudate $1 \mathrm{~h}$ after intake (Kumakura et al, 2004). Similarly, for haloperidol, a PET study in healthy volunteers showed $60-70 \%$ D2 receptor occupancy $3 \mathrm{~h}$ after administration (Nordstrom et al, 1992).

All tablets were over-encapsulated by the hospital pharmacy to ensure that both participants and experimenters could not compare or identify the drugs. Randomization was carried out by the hospital pharmacy. Each participant gave signed informed consent in which confidentiality, anonymity, and the opportunity to withdraw without penalty were assured. The study was approved by the Medical Ethics Committee of the Leiden University Medical Center and carried out according to the standards of the Declaration of Helsinki (Declaration of Helsinki, 2000). One participant was excluded from the analyses because of severe movement during scanning, and one participant dropped out after entering the scanner room. The final sample thus consisted of 53 participants (see Table 1 for subject variables in each group on age, body mass index, scores on psychoneuroticism as assessed with the Symptom Checklist-90 (Arrindell and Ettema, 1986); behavioral inhibition as assessed with the Behavioral Inhibition Behavioral Activation Scale Score (Carver and White, 1994); scores on impulsiveness using the Barratt Impulsiveness Scale (Patton et al, 1995); and sexual excitation and inhibition with Sexual Excitation and Sexual Inhibition Scales (Janssen et al, 2002); and finally, sexual arousal and anxiety using the Sexual Arousability Index (Hoon and Chambless, 1998)).

\section{Materials}

A backward-masking task was shown during fMRI scanning, which consisted of 160 trials with targets from four picture categories: sexual, emotionally negative, neutral, and fixation. Each trial started with a target picture with a duration of $26 \mathrm{~ms}$. (A duration of $33 \mathrm{~ms}$ is generally used in this paradigm (eg, Carlson et al, 2010; Childress et al, 2008), and is considered 'unseen'. However, we noticed in a pilot study that specifically sexual stimuli are still detectable at a $33 \mathrm{~ms}$ duration, whereas at $26 \mathrm{~ms}$, emotionally negative, neutral, and sexual pictures were all detected at below chance level.) The target was immediately followed by a mask, which was always a neutral picture (duration= $474 \mathrm{~ms}$ ). There was an inter-trial interval showing a gray fixation cross with a random duration between 0.5 and $2 \mathrm{~s}$ for jitter (see Figure 1). Under these conditions, participants see the masks, but the masked targets escape visual recognition and remain 'subconscious'. Neutral and emotionally negative pictures were selected from the International Affective Picture System (IAPS) (Lang et al, 2001), based on nine-point Likert SAM scales ratings of valence and arousal (neutral targets, $M \pm S D$ : 
Table I Means (M) and Standard Deviations (SD) of Subject Variables and ANOVA Results

\begin{tabular}{|c|c|c|c|c|c|}
\hline & Haloperidol, $n=18$ & Placebo, $n=17$ & Levodopa, $n=18$ & $F(2,52)$ & $p$-Value \\
\hline & $\mathbf{M} \pm \mathbf{S D}$ & $\mathbf{M} \pm \mathbf{S D}$ & $\mathbf{M} \pm \mathbf{S D}$ & & \\
\hline BMI & $22.86 \pm 2.37$ & $22.31 \pm 2.39$ & $22.31 \pm 1.65$ & 0.38 & 0.68 \\
\hline SCL-90 & $106.17 \pm 14.50$ & $106.00 \pm 13.69$ & $104.94 \pm 9.42$ & 0.05 & 0.95 \\
\hline Bas-Fun & $7.05 \pm 1.51$ & $7.88 \pm 1.97$ & $7.23 \pm 1.96$ & 0.96 & 0.39 \\
\hline Bas-Drive & $7.22 \pm 2.11$ & $7.17 \pm 1.67$ & $7.83 \pm 2.79$ & 0.42 & 0.66 \\
\hline Barratt-BIS & $65.56 \pm 6.64$ & $63.35 \pm 9.61$ & $67.18 \pm 10.98$ & 0.74 & 0.48 \\
\hline SES-total & $48.89 \pm 4.40$ & $49.71 \pm 6.34$ & $51.71 \pm 5.25$ & 1.26 & 0.29 \\
\hline SISI & $36.72 \pm 3.53$ & $38.76 \pm 2.86$ & $37.76 \pm 4.56$ & 1.32 & 0.28 \\
\hline
\end{tabular}

Note: BMI, body mass index; BIS-BAS total, Behavioral Inhibition Behavioral activation Scale Score; Barratt-BIS, Barratt Impulsiveness Scale; Bas R, Reward Responsiveness; SAI-Anx, Sexual Arousability Index-Anxiety; SAI-Aro, Sexual Arousability Index-Arousability; SCL-90, Symptom Checklist-90; SES-total, SISI and SIS2, Sexual Excitation and Sexual Inhibition Scales.

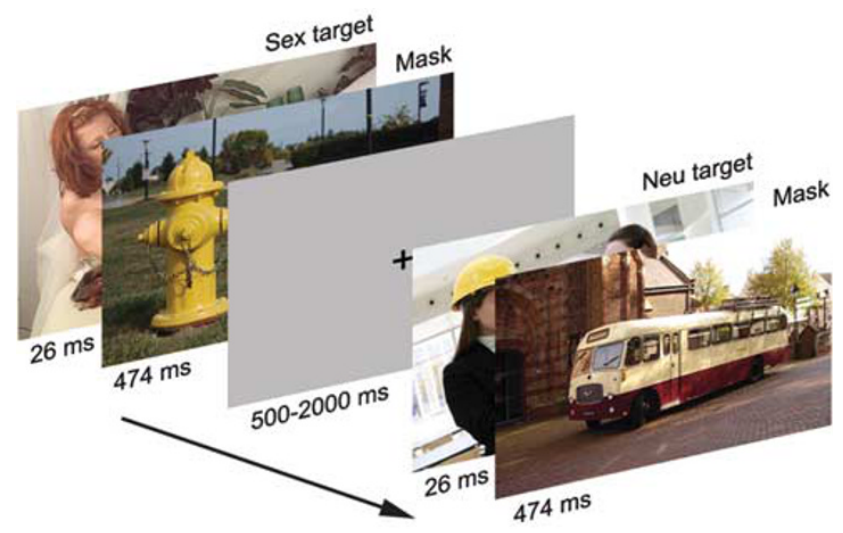

Figure I Backward-masking task. Note: 160 trials were randomly presented containing 26-ms targets from four categories (sex, emotionally negative, neutral, depicting humans, and a fixation) that were masked by 474-ms neutral (inanimate) pictures. Under these conditions, the targets escape conscious perception. The figure depicts an example of a sex trial and a neutral trial.

valence $=5.13 \pm 1.24$; arousal $=3.07 \pm 1.97$; emotionally negative targets: valence $=2.04 \pm 1.44$; arousal $=6.18 \pm 2.27$; neutral masks: valence $=5.04 \pm 1.30$; arousal $=3.03 \pm 1.86$ ) Neutral targets depicted humans, whereas the neutral masks were non-human (eg, an office cupboard). Emotionally negative targets depicted partly naked or naked humans, for example, mutilated bodies. Sexual targets were selected from picture sets previously used in sexology research and depicted partly naked or naked humans in a heterosexual erotic context (Spiering et al, 2003; Both et al, 2004).

All trials were randomly presented, and targets and masks within each category were randomly presented. The duration of the entire task was approximately $6 \mathrm{~min}$. Stimuli were presented in an $800 \times 600$ pixel resolution, back- projected on a screen located at the end of the scanner bore via an LCD projector located outside the scanner room. Subjects viewed stimuli on a screen through a mirror located on the head coil. Stimulus software (E-prime 1.2; Psychology Software Tools) was used for stimulus presentation.

To confirm that participants were not able to consciously detect the targets, a forced-choice category identification task was delivered after scanning. All trials were presented again, this time however, following each trial the participant had to indicate whether the target preceding the mask was a neutral, sexual, or emotionally negative picture.

\section{Scan protocol}

Imaging was carried out on a $3 \mathrm{~T}$ Philips Achieva MRI scanner (Philips, Best, The Netherlands), using an 8-channel SENSE head coil. A standard $T_{1}$-weighted structural volume and a high-resolution gradient echo planar image (EPI) scan were acquired for registration purposes. For fMRI during the backward-masking task, $T_{2}^{*}$-weighted gradient EPI sensitive to BOLD contrast were obtained in the axial direction (echo time $30 \mathrm{~ms}$, flip angle $80^{\circ}$, isotropic voxels of $2.75 \mathrm{~mm}, 0.25 \mathrm{~mm}$ slice gap, 38 slices, repetition time $2.2 \mathrm{~s})$.

\section{Procedure}

Upon arrival, the details of the experimental procedure were given, and informed consent was obtained. Because levodopa reaches peak-plasma concentration within $1 \mathrm{~h}$ after intake, whereas haloperidol reaches its peak $4 \mathrm{~h}$ after ingestion, participants always ingested two capsules, the first $4 \mathrm{~h}$ and the second $1 \mathrm{~h}$ before fMRI scanning, to ensure the concurrence of peak-plasma concentrations of both drugs during scanning. If a participant was assigned to the levodopa group, the first capsule contained placebo, 
whereas the second capsule contained levodopa. In the haloperidol group, the first capsule contained haloperidol, whereas the second capsule contained placebo. In the placebo group, a placebo was given twice (see also Pessiglione et al (2006) and Pleger et al (2009) for this administration protocol).

After ingestion of the first capsule, participants filled out questionnaires (see Table 1). They were allowed to read during the waiting period until ingestion of the second capsule. Exactly $1 \mathrm{~h}$ after ingesting the second capsule, the scanning started. Participants were instructed to watch attentively, and keep their eyes focused on the middle of the screen. After scanning, participants made the forced-choice categorization task on a computer. Next, an exit interview was administered in which participants were asked about their sentiments with regard to the experimental procedure. Finally, participants were thanked and paid for their participation and advised to refrain from alcohol and drug use the next $24 \mathrm{~h}$.

\section{Data Processing and Analysis}

FMRI data processing was carried out using FEAT (FMRI Expert Analysis Tool) Version 4.1, part of FSL (FMRIB's Software Library, www.fmrib.ox.ac.uk/fsl. The following pre-statistics processing was applied: motion correction (Jenkinson et al, 2002); non-brain removal (Smith, 2002); spatial smoothing using a Gaussian kernel of FWHM $8 \mathrm{~mm}$; grand-mean intensity normalization of the entire $4 \mathrm{D}$ data set by a single multiplicative factor; high-pass temporal filtering (Gaussian-weighted least-squares straight line fitting, with $\sigma=50.0 \mathrm{~s}$ ). Time-series statistical analysis was carried out with local autocorrelation correction (Woolrich et al, 2001). FMRI EPI data were registered to the highresolution EPI scan of each participant, which was registered to the individual T1-weighted structural scan, which was registered to the MNI-152 standard space template (Jenkinson and Smith, 2001; Jenkinson et al, 2002). Four explanatory variables (EVs) were included in the general linear model, representing the four target categories: neutral (Neu), sexual (Sex), emotionally negative (Neg), and fixation (Fix), each time-locked to the target onset, until mask offset. Each EV was convolved with a double gamma hemodynamic response function to account for the hemodynamic response. Contrasts of interest were Sex $v s$ Fix; Neg vs Fix; Sex $v s$ Neu; and Neg $v s$ Neu. For whole brain analysis, the images of contrasts of parameter estimates and corresponding variances were fed into a higher-level mixed-effects analysis, carried out with FLAME (FMRIB's Local Analysis of Mixed Effects) (Woolrich et al, 2004; Beckmann et al, 2003). To determine main task effects, irrespective of group assignment, a one-sample $t$-test was carried out. Whole brain $Z$ (Gaussianized $T$ ) statistic images were thresholded by an initial clusterforming threshold of $Z>2.3$ and a (corrected) cluster significance threshold of $p=0.05$. Then, the linear contrast (levodopa $>$ placebo $>$ haloperidol) was analyzed with independent ROI analyses, by masking the $Z$-stat images of the four task contrasts in the regions of interest before thresholding. Masks were binarized images of the NAcc, insula, dACC, thalamus, OFC, and caudate from the Harvard-Oxford Cortical and Subcortical Probability Atlas, set at a probability of $50 \%$. For the dACC, the subgenual part of the ACC was removed at MNI coordinate $y=32$ (McCormick et al, 2006). Thresholding was performed using GRF-theory-based maximum height thresholding, with a corrected significance threshold of $p=0.05$ (Worsley, 2001).

\section{RESULTS}

There was no relation between the medication the participants had received and the percentage that correctly guessed what they had received (Fisher's exact test $=8.29$, $p=0.16$ ), suggesting that blinding was adequate. Most participants reported no side effects $(n=41)$. Among the 12 participants who did have side effects, the most commonly reported ones were strange feeling in limbs, nausea, headache, dizziness, or strange vision. There were no differences in reported side effects across the three groups (Fisher's exact test $=3.98, p=0.42$ ).

The forced-choice category identification task delivered after scanning showed that mean forced-choice category identification was below chance level for all categories, indicating that participants were not able to discriminate the targets (see Table 2 for means and standard deviations). A repeated measures ANOVA was performed with Group (haloperidol $v s$ placebo $v s$ levodopa) as a between-subjects factor, Category (Neutral, Sex, Negative) as a withinsubjects factor, and task response as the dependent variable. This showed that there were no significant differences in mean correct identification between the categories $(\mathrm{F}(1.77$; $88.45)=0.29, p=0.72$, Greenhouse-Geisser corrected), no significant differences between the groups $(F(1,50)=0.08$, $p=0.92)$, and no significant interactions (F(3.53; $88.45)=0.39, p=0.79$, Greenhouse-Geisser corrected).

\section{Whole Brain Analysis}

See Figure 2 for the main effects of task in the contrast Sex $v s$ Fix. In this contrast, several clusters were significantly activated with peak values in the insula, OFC, and paracingulate gyrus and in more posterior regions, such as the (bi)lateral occipital cortex (see Table 3 for significant clusters and local maxima). The largest cluster had its peak in the insula and extended into frontal operculum cortices, caudate, thalamus, and bilateral NAcc. A cluster with its peak in the paracingulate gyrus encompassed local maxima

Table 2 Means (M) Percentage Correct Recognition and Standard Deviations (SD) on the Forced-Choice Category Identification Task

\begin{tabular}{cllllll}
\hline & \multicolumn{9}{c}{ Targets } \\
\cline { 2 - 7 } & \multicolumn{2}{c}{ Neutral } & \multicolumn{2}{c}{ Sexual } & \multicolumn{2}{c}{ Negative } \\
\hline Group & M & SD & M & SD & M & SD \\
Haloperidol & 32.22 & 9.27 & 35.00 & 9.70 & 35.28 & 10.06 \\
Placebo & 32.94 & 7.51 & 34.41 & 6.34 & 32.94 & 10.62 \\
Levodopa & 34.72 & 9.47 & 34.16 & 6.24 & 33.33 & 9.85 \\
Total & 33.30 & 8.71 & 34.52 & 7.48 & 33.86 & 10.03 \\
\hline
\end{tabular}


a

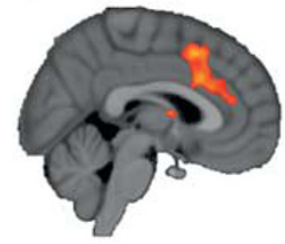

b

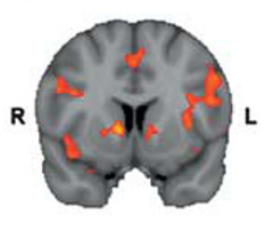

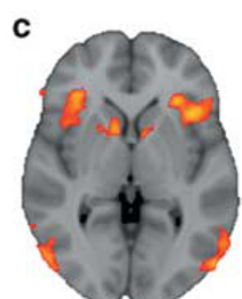

Figure 2 Main effect of the contrast Sex> Fix. Note: (a) Coronal, (b) sagittal, and (c) axial view of clusters of voxels $(Z>2.3, p=0.05$, clustercorrected) when contrasting Sex vs Fix (MNI coordinates, $x, y, z=3,9,1$ ). Intensity values in this thresholded zstat map range from 2.3 (red) to 5 (yellow). Voxel size $=2 \mathrm{~mm}^{3}$ in standard space.

in the dACC. The contrast Sex vs Neu showed significant clusters (see Table 3) in OFC, inferior lateral occipital cortex, and inferior frontal gyrus, with local maxima in the insula and caudate encompassing NAcc. In the contrast Neg $v s$ Fix, two significant clusters were found: one in the lingual gyrus and one that extended from frontal operculum cortex into the left insular cortex. No significant clusters were found in the contrast Neg $v s$ Neu. The results from the latter two contrasts suggest that the (masked) sexual stimuli were specific in inducing activation in reward-related brain areas.

\section{ROI Analysis}

With independent ROI analyses, the linear contrast Group (levodopa $>$ placebo $>$ haloperidol) was analyzed, to test the hypothesis that subconscious stimulation of the reward system would be enhanced by DA activation and suppressed by DA inhibition. In Sex $v s$ Fix, this contrast was significant $(p<0.05$, voxel-corrected) in both bilateral NAcc and dACC (see Figure 3). In Sex vs Neu, the linear contrast was significant in the right NAcc, but not in other ROIs. There were no significant differences in activation in the ROIs in the remaining contrasts.

\section{DISCUSSION}

This study was aimed at investigating the modulatory effects of DA on the processing of subconsciously perceived sexual stimuli. Our findings showed that DA stimulates activity in key brain areas of the reward system, the NAcc and AACC, in response to subconsciously perceived sexual stimuli. These results provide the first evidence for pharmacological modulation of implicit sexual reward processes, pointing at the possibility for DA to affect sexual motivation at its earliest onset, that is, outside awareness. These findings, again, emphasize the sensitivity of the brain to signals for sexual reward, even when these are not detected consciously, consistent with other reports showing activations in the ventral striatum (Childress et al, 2008), thalamus, and ACC (Gillath and Canterberry, 2011) during the subliminal presentation of erotic stimuli.

Most pronounced were the effects of DA in the NAcc and the dACC, where a linear association was found dependent on the administration of haloperidol, placebo, or levodopa. The NAcc plays a central role in the reward circuit and its activity has frequently been related to processing of secondary rewards, such as money (Assadi et al, 2009;
Kelley, 2004; McClure et al, 2003; Pessiglione et al, 2006; Pleger et al, 2009), and also of sexual stimuli (Walter et al, 2008). The dACC, as a rule of thumb, has been associated with the more cognitive aspects of stimulus processing, and ventral ACC more with emotional processes; nonetheless, the dACC is a key contributor to emotional processing (Etkin et al, 2011). The roles that are attributed to the dACC are numerous, but the common denominator is its role in various aspects of apt decision-making, from initial sensory perception to motor preparation (Assadi et al, 2009). The dACC is connected to the NAcc and ventral putamen, which together with its DA system is suggested to be involved in the evaluation and execution of decision-making. The dACC participates in motivation ('wanting') through mobilization of resources, and initiation of goal-directed behaviors, through its projections to the motor area, and the periaquaductal gray matter, the latter also known for generating sexual behaviors (Assadi et al, 2009; Lonstein and Stern, 1998). dACC is associated with autonomic modulation of heart rate and pupil dilation (Critchley et al, 2003, 2005). Furthermore, dACC abnormalities have been found in obsessive-compulsive disorders, schizophrenia, and addiction (Yucel et al, 2007a, b, c). DA dysregulation in dACC-NAcc networks are suggested to impair accurate decision-making, for instance, to oversee the costs of pursuing deviant impulses and the inability to learn from previous mistakes (Assadi et al, 2009).

Interestingly, the responses to sexual stimuli in our task, regardless of group assignment, also showed significant activations in bilateral anterior insula, which together with the dACC form the 'salience network' during no task conditions (Menon and Uddin, 2010). According to the recent model by Menon and Uddin (2010), the insula functions as an integral hub that detects salient stimuli, switches between other large-scale networks to facilitate access to attention and working memory, it modulates autonomic responses to those stimuli, and facilitates rapid access to the motor system through its coupling with the ACC. The activation of the salience network only during processing of sexual cues indicates their facilitated processing, which may have prompted autonomic or motor responses. Implicit processing of erotic stimuli has shown to evoke early autonomic reactions and motor preparation in previous studies (Janssen et al, 2000; Both et al, 2008b).

DA neurons are also known to respond to aversive states and stimuli, although with excitation in some and inhibition in other DA neurons (Bromberg-Martin et al, 2010). Animal studies suggest that in response to aversive states, after a brief initial increase, DA is decreased and then released when aversive stimuli are removed (Budygin et al, 2012; Cabib and Puglisi-Allegra, 2012). However, in this study, no activation in the ventral striatum was detected in response to the masked emotionally negative stimuli, even when lowering the threshold to uncorrected activation levels. Surprisingly, we also did not find amygdala activation in response to the masked emotionally negative pictures. Childress et al (2008) also did not detect any significant differences in activation between negative and neutral pictures using similar aversive pictures in a similar backward masking paradigm. They suggested that the lack of effects might be due to large inter-subject variability related to other variables, such as anxiety proneness. Nonetheless, 
Table 3 Cluster List of Significant Main Effects of Task

\begin{tabular}{|c|c|c|c|c|c|c|c|}
\hline Region & L/R & Cluster size & $\mathbf{z}$ & $x$ & $y$ & $\mathbf{z}$ & $p$-Value \\
\hline \multicolumn{8}{|l|}{ SEX $>$ FIX } \\
\hline Insula & $\mathrm{L}$ & 2765 & 4.66 & -30 & 28 & 4 & $<0.0001$ \\
\hline Caudate & $\mathrm{L}$ & & 3.29 & -10 & 8 & 4 & \\
\hline Caudate & $\mathrm{R}$ & & 4.34 & 12 & 8 & 2 & \\
\hline Thalamus & $\mathrm{R}$ & & 4.13 & 4 & -2 & 8 & \\
\hline Nucleus accumbens & L & & 2.68 & -8 & 16 & -4 & \\
\hline Nucleus accumbens & $\mathrm{R}$ & & 2.74 & 10 & 16 & -4 & \\
\hline Inferior lateral occipital cortex & $\mathrm{L}$ & 1317 & 4.66 & -54 & -76 & 6 & $<0.0001$ \\
\hline Temporal occipital fusiform cortex & $\mathrm{L}$ & & 4.3 & -42 & -56 & -22 & \\
\hline Insula & $\mathrm{R}$ & & 3.35 & 42 & 14 & 0 & \\
\hline Inferior frontal gyrus & $\mathrm{R}$ & & 3.35 & 58 & 34 & 6 & \\
\hline Paracingulate gyrus & & $110 \mid$ & 4.52 & 4 & 16 & 46 & $<0.000$ I \\
\hline Dorsal anterior cingulate & & & 4.04 & 2 & 18 & 30 & \\
\hline Dorsal anterior cingulate & & & 3.71 & 2 & 26 & 26 & \\
\hline Dorsal anterior cingulate & & & 3.33 & 6 & 14 & 24 & \\
\hline Inferior lateral occipital cortex & $\mathrm{R}$ & 805 & 4.57 & 54 & -74 & 12 & 0.0007 \\
\hline Superior lateral occipital cortex & $\mathrm{R}$ & & 4.03 & 46 & -66 & 18 & \\
\hline Middle temporal gyrus & $\mathrm{R}$ & & 2.82 & 66 & -54 & 2 & \\
\hline Supramarginal gyrus & $\mathrm{R}$ & 517 & 3.71 & 36 & -46 & 34 & 0.01 \\
\hline Superior lateral occipital cortex & $\mathrm{L}$ & & 3.57 & -28 & -60 & 50 & \\
\hline \multicolumn{8}{|l|}{$S E X>N E U$} \\
\hline Inferior lateral occipital cortex & $\mathrm{L}$ & 3715 & 5.51 & -54 & -72 & 12 & $<0.000$ । \\
\hline Inferior temporal cortex & L & & 4.11 & -44 & -50 & -26 & \\
\hline Temporal occipital fusiform cortex & $\mathrm{L}$ & & 4.08 & -42 & -48 & -20 & \\
\hline Orbitofrontal cortex & $\mathrm{L}$ & 2488 & 4.86 & -30 & 28 & 2 & $<0.000$ I \\
\hline Insula & $\mathrm{L}$ & & 4.66 & -36 & 20 & 2 & \\
\hline Caudate & $\mathrm{R}$ & & 4.21 & 10 & 10 & 4 & \\
\hline Caudate & L & & 3.93 & -8 & 4 & 6 & \\
\hline Nucleus accumbens & $\mathrm{R}$ & & 3.19 & 8 & 12 & -4 & \\
\hline Nucleus accumbens & $\mathrm{L}$ & & 3.54 & -8 & 16 & -4 & \\
\hline Temporal occipital fusiform cortex & $\mathrm{R}$ & $|76|$ & 4.73 & 46 & -52 & -20 & $<0.000$ । \\
\hline Inferior lateral occipital cortex & $\mathrm{R}$ & & 4.54 & 56 & -70 & -2 & \\
\hline Orbitofrontal cortex & $\mathrm{R}$ & 1626 & 4.47 & 36 & 28 & -2 & $<0.000$ । \\
\hline Frontal operculum cortex & $\mathrm{R}$ & & 4.3 & 46 & 20 & 22 & \\
\hline Inferior frontal gyrus, triangularis & $\mathrm{R}$ & & 3.79 & 42 & 24 & 16 & \\
\hline Inferior frontal gyrus, opercularis & $\mathrm{L}$ & 895 & 4.22 & -44 & 10 & 20 & $<0.000$ I \\
\hline Precentral gyrus & L & & 4.2 & -42 & 4 & 34 & \\
\hline Middle frontal gyrus & $\mathrm{L}$ & & 4.05 & -44 & 6 & 38 & \\
\hline
\end{tabular}


Table 3 Continued

\begin{tabular}{|c|c|c|c|c|c|c|c|}
\hline Region & L/R & Cluster size & $\mathbf{Z}$ & $x$ & $y$ & $\mathbf{z}$ & p-Value \\
\hline \multicolumn{8}{|l|}{$N E G>F I X$} \\
\hline Lingual gyrus & L & 998 & 3.76 & -4 & -82 & -6 & $<0.000$ I \\
\hline Occipital pole & L & & 3.5 & -2 & -92 & 10 & \\
\hline Intracalcarine cortex & L & & 3.24 & -2 & -88 & 4 & \\
\hline Inferior frontal gyrus, opercularis & L & 464 & 3.72 & -46 & 16 & 4 & 0.04 \\
\hline
\end{tabular}

Note: $Z>2.3, p=0.05$, cluster-corrected. $L / R=$ left/right in the brain; voxel size is $2 \mathrm{~mm}$ isotropic.
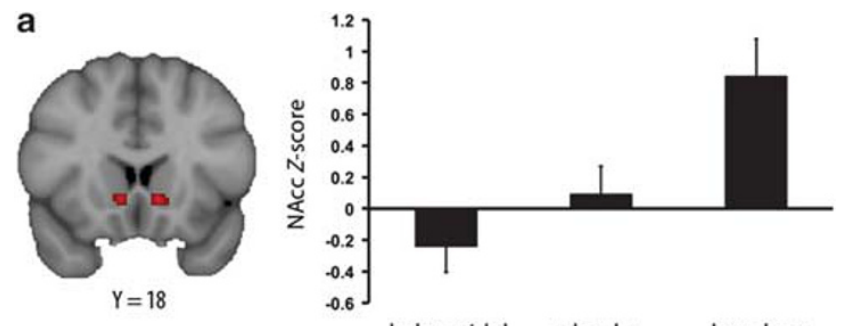

b

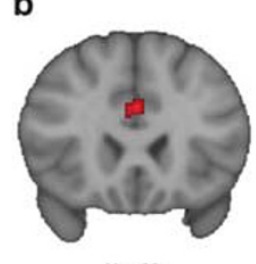

$Y=22$

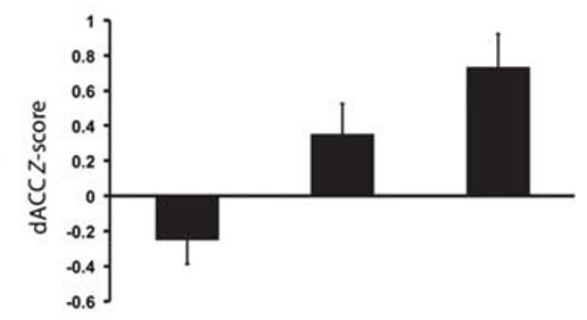

Figure 3 Contrasting Sex> Fix in the nucleus accumbens (NAcc) and dorsal anterior cingulate cortex (dACC). Note: The thresholded zstat maps depict coronal view of voxels (voxel size $=2 \mathrm{~mm}^{3}$ in standard space) significantly more active in the linear contrast levodopa $>$ placebo $>$ haloperidol (voxel-wise corrected, $p<0.05$ ) in the (a) NAcc and (b) dACC. Plots depict $Z$-values (mean group \pm SEM) from all groups extracted from these voxels.

several studies using masked fearful faces did report amygdala activation (eg, Carlson et al, 2009). Consciously perceived faces appear to evoke somewhat stronger amygdala activation than complex aversive IAPS pictures, even though the latter are rated as more arousing (Britton et al, 2006). Faces are less complex than aversive pictures, which may facilitate masked presentations. However, the pictures that were selected for this study were similar in complexity to the sexual pictures. It could be speculated that the processing of masked emotional stimuli might be facilitated when it comes to emotionally positive stimuli compared with emotionally negative stimuli. If the general default tendency to approach is greater than the tendency to avoid (Cacioppo et al, 1997; Cacioppo and Gardner, 1999), the first might be more responsive to weak positive stimuli, such as masked sexual pictures, whereas the latter tendency might be more likely evoked by more intense aversive stimuli.

Regretfully, we did not investigate whether DA-dependent increased activations in regions thought to regulate incentive salience was related to increases in desire or wanting, which could, for instance, be assessed by behavioral approach tendencies. There is, however, evidence for enhanced tendencies to approach sexual stimuli following levodopa administration (Both et al, 2005). Future studies ideally should incorporate such measures when investigating the effects of subliminal rewarding stimuli. Furthermore, another component of reward, that is, associative learning is very relevant when studying hypersexuality (Klucken et al, 2009; Both et al, 2008a, b). With DA modulation, it could be investigated how DA affects the incentive reward of neutral stimuli paired with rewarding ones. Possibly, individual differences in DA sensitivity (Ben Zion et al, 2006), in combination with frequent exposure to sexual cues and reinforcement processes, might explain the initiation of aberrant sexual desires.

The DA-dependent 'running start' of the reward system, well before the motivational state is consciously experienced as wanting, might explain the struggle to manage the pull of rewards, as evident in individuals with compulsive rewardseeking behaviors such as addictions and hypersexuality. These subconscious processes also might play a role in Parkinson's patients who develop an increased sexual preoccupation while using dopaminergic medication or in schizophrenic patients whose sexual desire decreases during antipsychotic therapy. Future investigations should target the influence of DA on decision-making subsequent to implicit sexual reward processing, and ideally should include clinical hypersexual populations.

\section{ACKNOWLEDGEMENTS}

We are grateful to Ilya Veer and Michiel de Ruiter for their helpful comments during the data analysis and Olga Teutler for helping with the data collection. SB and NO were supported by a grant from the European Society for Sexual Medicine (ESSM). RS and SR were supported by a grant of the Netherlands Organization for Scientific Research (NWO).

\section{DISCLOSURE}

The authors declare that, except for income received from their primary employer, no financial support or 
compensation has been received from any individual or corporate entity over the past 3 years for research or professional service and there are no personal financial holdings that could be perceived as constituting a potential conflict of interest.

\section{REFERENCES}

Agmo A (1999). Sexual motivation - an inquiry into events determining the occurrence of sexual behavior. Behav Brain Res 105: 129-150.

Arrindell WA, Ettema JHM (1986) SCL-90. Handleiding bij een Multidimensionele Psychopathologie-Indicator. Swets \& Zeitlinger BV: Lisse.

Assadi SM, Yucel M, Pantelis C (2009). Dopamine modulates neural networks involved in effort-based decision-making. Neurosci Biobehav Rev 33: 383-393.

Beckmann CF, Jenkinson M, Smith SM (2003). General multilevel linear modeling for group analysis in FMRI. NeuroImage 20: 1052-1063.

Ben Zion IZ, Tessler R, Cohen L, Lerer E, Raz Y, Bachner-Melman $\mathrm{R}$ et al (2006). Polymorphisms in the dopamine $\mathrm{D} 4$ receptor gene (DRD4) contribute to individual differences in human sexual behavior: desire, arousal and sexual function. Mol Psychiatry 11: 782-786.

Berridge KC, Robinson TE (1998). What is the role of dopamine in reward: hedonic impact, reward learning, or incentive salience? Brain Res Brain Res Rev 28: 309-369.

Berridge KC, Robinson TE (2003). Parsing reward. Trends Neurosci 26: 507-513.

Both S, Everaerd W, Laan E (2007). Desire emerges from excitement: a psychophysiological perspective on sexual motivation. In: Janssen E (ed). The Psychophysiology of Sex. Indiana University Press: Bloomington, IN. pp 327-339.

Both S, Everaerd W, Laan E, Gooren L (2005). Effect of a single dose of levodopa on sexual response in men and women. Neuropsychopharmacology 30: 173-183.

Both S, Laan E, Spiering M, Nilsson T, Oomens S, Everaerd W (2008a). Appetitive and aversive classical conditioning of female sexual response. J Sex Med 5: 1386-1401.

Both S, Spiering M, Everaerd W, Laan E (2004). Sexual behavior and responsiveness to sexual stimuli following laboratoryinduced sexual arousal. J Sex Res 41: 242-258.

Both S, Spiering M, Laan E, Belcome S, van den Heuvel B, Everaerd W (2008b). Unconscious classical conditioning of sexual arousal: evidence for the conditioning of female genital arousal to subliminally presented sexual stimuli. J Sex Med 5: 100-109.

Britton JC, Taylor SF, Sudheimer KD, Liberzon I (2006). Facial expressions and complex IAPS pictures: common and differential networks. NeuroImage 31: 906-919.

Bromberg-Martin ES, Matsumoto M, Hikosaka O (2010). Dopamine in motivational control: rewarding, aversive, and alerting. Neuron 68: 815-834.

Budygin EA, Park J, Bass CE, Grinevich VP, Bonin KD, Wightman RM (2012). Aversive stimulus differentially triggers subsecond dopamine release in reward regions. Neuroscience 201: 331-337.

Cabib S, Puglisi-Allegra S (2012). The mesoaccumbens dopamine in coping with stress. Neurosci Biobehav Rev 36: 79-89.

Cacioppo JT, Gardner WL (1999). Emotion. Annu Rev Psychol 50: 191-214.

Cacioppo JT, Gardner WL, Berntson GG (1997). Beyond bipolar conceptualizations and measures: the case of attitudes and evaluative space. Pers Soc Psychol Rev 1: 3-25.

Carlson JM, Greenberg T, Mujica-Parodi LR (2010). Blind rage? Heightened anger is associated with altered amygdala responses to masked and unmasked fearful faces. Psychiatry Res 182: 281-283.
Carlson JM, Reinke KS, Habib R (2009). A left amygdala mediated network for rapid orienting to masked fearful faces. Neuropsychologia 47: 1386-1389.

Carver CS, White TL (1994). Behavioral inhibition, behavorial activation, and affective responses to impending reward and punishment: The BIS/BAS scales. J Person Soc Psychol 67: 319-333.

Childress AR, Ehrman RN, Wang Z, Li Y, Sciortino N, Hakun J et al (2008). Prelude to passion: limbic activation by 'unseen' drug and sexual cues. PLoS One 3: e1506.

Critchley HD, Mathias CJ, Josephs O, O’Doherty J, Zanini S, Dewar BK et al (2003). Human cingulate cortex and autonomic control: converging neuroimaging and clinical evidence. Brain 126: 2139-2152.

Critchley HD, Tang J, Glaser D, Butterworth B, Dolan RJ (2005). Anterior cingulate activity during error and autonomic response. NeuroImage 27: 885-895.

Declaration of Helsinki 52nd WMA General Assembly. Declaration of Helsinki: Edinburgh, UK; (2000).

Etkin A, Egner T, Kalisch R (2011). Emotional processing in anterior cingulate and medial prefrontal cortex. Trends Cogn Sci 15: 85-93.

Evans AH, Strafella AP, Weintraub D, Stacy M (2009). Impulsive and compulsive behaviors in Parkinson's disease. Mov Disord 24: $1561-1570$.

Gillath O, Canterberry M (2011). Neural correlates of exposure to subliminal and supraliminal sexual cues. Soc Cogn Affect Neurosci; doi:10.1093/scan/nrs065.

Haber SN, Knutson B (2010). The reward circuit: linking primate anatomy and human imaging. Neuropsychopharmacology 35: 4-26.

Hoon EF, Chambless D (1998). Sexual Arousability Inventory and Sexual Arousability Inventory-expanded. In: Davis CM, Yarber WL, Bauserman R, Schreer G, Davis SL (eds). Handbook of Sexuality-Related Measures. Sage Publications: Thousand Oaks, CA, pp 71-74.

Janssen E, Everaerd W, Spiering M, Janssen J (2000). Automatic cognitive processes and the appraisal of sexual stimuli: towards an information processing model of sexual arousal. J Sex Res 37: 8-23.

Janssen E, Vorst H, Finn P, Bancroft J (2002). The Sexual Inhibition (SIS) and Sexual Excitation (SES) Scales: I. Measuring sexual inhibition and excitation proneness in men. J Sex Res 39: 114-126.

Jenkinson M, Bannister P, Brady M, Smith S (2002). Improved optimization for the robust and accurate linear registration and motion correction of brain images. Neurolmage 17: 825-841.

Jenkinson M, Smith S (2001). A global optimisation method for robust affine registration of brain images. Med Image Anal 5: $143-156$.

Kelley AE (2004). Ventral striatal control of appetitive motivation: role in ingestive behavior and reward-related learning. Neurosci Biobehav Rev 27: 765-776.

Khor SP, Hsu A (2007). The pharmacokinetics and pharmacodynamics of levodopa in the treatment of Parkinson's disease. Curr Clin Pharmacol 2: 234-243.

Klucken T, Schweckendiek J, Merz CJ, Tabbert K, Walter B, Kagerer S et al (2009). Neural activations of the acquisition of conditioned sexual arousal: effects of contingency awareness and sex. J Sex Med 6: 3071-3085.

Kringelbach ML, Berridge KC (2009). Towards a functional neuroanatomy of pleasure and happiness. Trends Cogn Sci 13: 479-487.

Kumakura Y, Danielsen EH, Reilhac A, Gjedde A, Cumming P (2004). Levodopa effect on $\left[{ }^{18} \mathrm{~F}\right]$ fluorodopa influx to brain: normal volunteers and patients with Parkinson's disease. Acta Neurol Scand 110: 188-195. 
Lang PJ, Bradley MM, Cuthbert BN (2001). International Affective Picture System (IAPS): Instruction Manual and Affective Ratings. The Center for Research in Psychophysiology, University of Florida: Florida.

Liem-Moolenaar M, Gray FA, de Visser SJ, Franson KL, Schoemaker RC, Schmitt JA et al (2010). Psychomotor and cognitive effects of a single oral dose of talnetant (SB223412) in healthy volunteers compared with placebo or haloperidol. J Psychopharmacol 24: 73-82.

Lonstein JS, Stern JM (1998). Site and behavioral specificity of periaqueductal gray lesions on postpartum sexual, maternal, and aggressive behaviors in rats. Brain Res 804: 21-35.

McClure SM, Berns GS, Montague PR (2003). Temporal prediction errors in a passive learning task activate human striatum. Neuron 38: 339-346.

McCormick LM, Ziebell S, Nopoulos P, Cassell M, Andreasen NC, Brumm M (2006). Anterior cingulate cortex: an MRI-based parcellation method. NeuroImage 32: 1167-1175.

Menon V, Uddin LQ (2010). Saliency, switching, attention and control: a network model of insula function. Brain Struct Funct 214: 655-667.

Midha KK, Chakraborty BS, Ganes DA, Hawes EM, Hubbard JW, Keegan DL et al (1989). Intersubject variation in the pharmacokinetics of haloperidol and reduced haloperidol. J Clin Psychopharmacol 9: 98-104.

Nordstrom AL, Farde L, Halldin C (1992). Time course of D2dopamine receptor occupancy examined by PET after single oral doses of haloperidol. Psychopharmacology (Berl) 106: 433-438.

O'Sullivan SS, Wu K, Politis M, Lawrence AD, Evans AH, Bose SK et al (2011). Cue-induced striatal dopamine release in Parkinson's disease-associated impulsive-compulsive behaviours. Brain 134: 969-978.

Patton JH, Stanford MS, Barratt ES (1995). Factor structure of the Barratt impulsiveness scale. J Clin Psychol 51: 768-774.

Pessiglione M, Seymour B, Flandin G, Dolan RJ, Frith CD (2006). Dopamine-dependent prediction errors underpin reward-seeking behaviour in humans. Nature 442: 1042-1045.

Pleger B, Ruff CC, Blankenburg F, Kloppel S, Driver J, Dolan RJ (2009). Influence of dopaminergically mediated reward on somatosensory decision-making. PLoS Biol 7: e1000164.

Robinson TE, Berridge KC (1993). The neural basis of drug craving: an incentive-sensitization theory of addiction. Brain Res Brain Res Rev 18: 247-291.

Rosen RC, Riley A, Wagner G, Osterloh IH, Kirkpatrick J, Mishra A (1997). The international index of erectile function (IIEF): a multidimensional scale for assessment of erectile dysfunction. Urology 49: 822-830.
Sagar KA, Smyth MR (2000). Bioavailability studies of oral dosage forms containing levodopa and carbidopa using column-switching chromatography followed by electrochemical detection. Analyst 125: 439-445.

Sheehan DV, Lecrubier Y, Sheehan KH, Amorim P, Janavs J, Weiller E et al (1998). The Mini-International Neuropsychiatric Interview (MINI): the development and validation of a structured diagnostic psychiatric interview for DSM-IV and ICD-10. J Clin Psychiatry 59: 22-33.

Singer B, Toates FM (1987). Sexual motivation. J Sex Res 23: 481-501.

Smith SM (2002). Fast robust automated brain extraction. Hum Brain Mapp 17: 143-155.

Spiering M, Everaerd W, Janssen E (2003). Priming the sexual system: implicit versus explicit activation. J Sex Res 40: 134-145.

Stewart J (1995). How does incentive motivational theory apply to sexual behavior?. In: Bancroft J (ed). The Pharmacology of Sexual Function and Dysfunction. Elsevier Science BV: Amsterdam. pp 3-11.

Walter M, Bermpohl F, Mouras H, Schiltz K, Tempelmann C, Rotte $M$ et al (2008). Distinguishing specific sexual and general emotional effects in fMRI-subcortical and cortical arousal during erotic picture viewing. NeuroImage 40: 1482-1494.

Wilde GJS (1963). Neurotische labiliteit gemeten volgens de vragenlijstmethode. van Rossen: Amsterdam, 1963.

Woolrich MW, Behrens TE, Beckmann CF, Jenkinson M, Smith SM (2004). Multilevel linear modelling for FMRI group analysis using Bayesian inference. NeuroImage 21: 1732-1747.

Woolrich MW, Ripley BD, Brady M, Smith SM (2001). Temporal autocorrelation in univariate linear modeling of FMRI data. NeuroImage 14: 1370-1386.

Worsley KJ (2001). Statistical analysis of activation images. In: Jezzard P, Matthews PM, Smith SM (eds). Functional MRI: An Introduction to Methods. Oxford University Press Inc.: New York, NY. pp 251-270.

Yucel M, Brewer WJ, Harrison BJ, Fornito A, O’Keefe GJ, Olver J et al (2007a). Anterior cingulate activation in antipsychoticnaive first-episode schizophrenia. Acta Psychiatr Scand 115: 155-158.

Yucel M, Harrison BJ, Wood SJ, Fornito A, Wellard RM, Pujol J et al (2007b). Functional and biochemical alterations of the medial frontal cortex in obsessive-compulsive disorder. Arch Gen Psychiatry 64: 946-955.

Yucel M, Lubman DI, Harrison BJ, Fornito A, Allen NB, Wellard RM et al (2007c). A combined spectroscopic and functional MRI investigation of the dorsal anterior cingulate region in opiate addiction. Mol Psychiatry 12: 611, 691-702. 